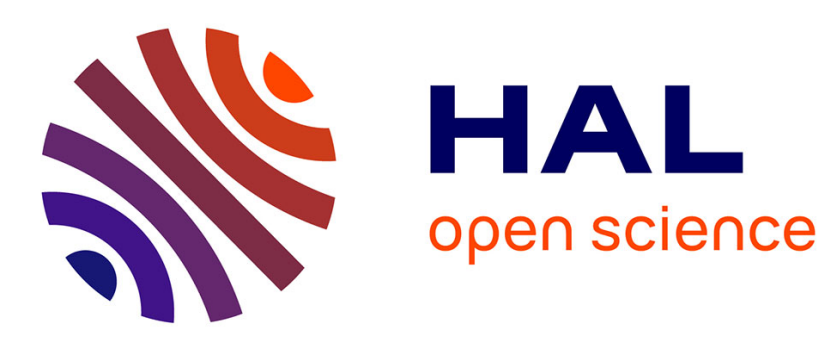

\title{
Effect of Ions on Water Dynamics in Dilute and Concentrated Aqueous Salt Solutions
}

\author{
Damien Laage, Guillaume Stirnemann
}

\section{To cite this version:}

Damien Laage, Guillaume Stirnemann. Effect of Ions on Water Dynamics in Dilute and Concentrated Aqueous Salt Solutions. Journal of Physical Chemistry B, 2019, 123 (15), pp.3312-3324. 10.1021/acs.jpcb.9b01053 . hal-02302089

\section{HAL Id: hal-02302089 \\ https://hal.science/hal-02302089}

Submitted on 1 Oct 2019

HAL is a multi-disciplinary open access archive for the deposit and dissemination of scientific research documents, whether they are published or not. The documents may come from teaching and research institutions in France or abroad, or from public or private research centers.
L'archive ouverte pluridisciplinaire HAL, est destinée au dépôt et à la diffusion de documents scientifiques de niveau recherche, publiés ou non, émanant des établissements d'enseignement et de recherche français ou étrangers, des laboratoires publics ou privés. 


\title{
On the Effect of Ions on Water Dynamics in Dilute and Concentrated Aqueous Salt Solutions
}

\author{
Damien Laage ${ }^{\dagger}$ and Guillaume Stirnemann*,‡ \\ PASTEUR, Département de chimie, École normale supérieure, PSL University, Sorbonne \\ Université, CNRS, 75005 Paris, France, and CNRS Laboratoire de Biochimie Théorique, \\ Institut de Biologie Physico-Chimique, PSL University, Sorbonne Paris Cité, 13 rue Pierre \\ et Marie Curie, 75005, Paris, France \\ E-mail: stirnemann@ibpc.fr
}

\section{Abstract}

Aqueous ionic solutions are ubiquitous in chemistry and in biology. Experiments show that ions affect water dynamics, but a full understanding of several questions remains needed: why some salts accelerate water dynamics while others slow it down, why the effect of a given salt can be concentration dependent, whether the effect of ions is rather local or more global. Numerical simulations are particularly suited to disentangle these different effects, but current force fields suffer from limitations and often lead to a poor description of dynamics in several aqueous salt solutions. Here, we develop an improved classical force field for the description of alkali halides which yields dynamics in excellent agreement with experimental measurements for water reorientational and translational dynamics. These simulations are analyzed with an extended jump model, which allows to compare the effects of ions on local hydrogen-bond exchange dynamics and on more global properties

\footnotetext{
${ }^{*}$ To whom correspondence should be addressed

†PASTEUR, Département de chimie, École normale supérieure, PSL University, Sorbonne Université, CNRS, 75005 Paris, France

${ }^{\ddagger}$ CNRS Laboratoire de Biochimie Théorique, Institut de Biologie Physico-Chimique, PSL University, Sorbonne Paris Cité, 13 rue Pierre et Marie Curie, 75005, Paris, France
}

like viscosity. Our results unambiguously show that the ion-induced changes in water dynamics are usually mostly due to a local effect on the hydrogen-bond exchange dynamics; in contrast, the change in viscosity leads to a smaller effect, which governs the retardation only for a minority of salts and at high concentrations. We finally show how the respective importance of these two effects can be directly determined from experimental measurements alone, thus providing guidelines for the selection of an electrolyte with specific dynamical properties.

\section{Introduction}

Liquid water most often contains dissolved salts that play a central role in many chemical and biological processes. These ions alter water structure and dynamics, which can be studied by a variety of experimental and simulation techniques. ${ }^{1}$ While understanding the ioninduced change in water dynamical properties is key to explain critical aspects of the involved processes, rationalizing the effects of a given salt is not straightforward because (i) these effects are very sensitive to the nature of the cation and of the anion; ${ }^{1}$ (ii) they exhibit a marked concentration-dependence; ${ }^{2}$ (iii) different techniques probe distinct aspects of water dynamics that are not necessarily affected in the 
same way and that correspond to very different molecular motions. ${ }^{3}$ Therefore, despite recent advances, important questions remain not fully solved: why do some salts accelerate dynamics while others retard it? Why is the effect of a given salt concentration-dependent? Is this effect local (i.e., restricted to the first- or secondhydration shell) or rather due to a more global property of the solution, e.g. analogous to that of pressure, as suggested in some prior studies ${ }^{4}$ ?

Numerical simulations appear as a promising tool to disentangle these different aspects. We have recently studied the water reorientation dynamics in various ionic hydration shells and solutions ${ }^{5-7}$ using classical molecular dynamics (MD) simulations analyzed in the framework of the extended jump model (EJM). ${ }^{3,8}$ Two mechanisms contribute to the reorientation of water $\mathrm{OH}$ groups ${ }^{3,8}$ on the picosecond (ps) timescale, which is measured experimentally by NMR or ultrafast infrared spectroscopy: the jump exchange of hydrogen-bond (HB) acceptors, and the diffusive reorientation of the frame formed by an intact $\mathrm{HB}$ axis between HB exchanges, which scales with the solution viscosity. We had shown that reorientation around dilute solutes, including ions ${ }^{5-7}$ but also organic compounds with various properties (hydrophobic, ${ }^{9}$ hydrophilic, ${ }^{10}$ or amphiphilic ${ }^{9,10}$ ) was slowed down or accelerated mainly because of changes in the jump $\mathrm{HB}$ exchange time. While in the vast majority of cases, HB exchanges remained the main contribution to the water reorientation dynamics, we had suggested that the slowdown observed in all ionic aqueous solutions at higher concentrations contained a non-specific contribution, which originates from an increasing frame reorientation time (correlated with an increase in viscosity), and which can become dominant for some salts. ${ }^{7}$ Similar observations had been made on other, non-ionic solutions. ${ }^{11}$

Our previous studies on ions have been limited to dilute halides, ${ }^{5-7}$ and to dilute and concentrated sodium sulfate and sodium perchlorate solutions. ${ }^{7}$ Very recently, simulations of sodium and potassium thiocyanate solutions from dilute conditions up to very high concentrations were analyzed using the same approach we originally applied to other solutions. ${ }^{12,13}$
This study proposed a general picture, where in all ionic solutions, reorientation is slowed down at high concentration because of a slowed frame reorientation (linked to viscosity). While we had already highlighted the importance of this frame reorientation in some specific solutions, ${ }^{7}$ we had shown that it did not apply to all solutions.

Here our goal is to clarify this issue and gain a molecular understanding of the different ion effects on the dynamics of water both in dilute and concentrated aqueous solutions. We extend our initial approach to a broad range of alkali halide solutions, from dilute conditions up to $5 \mathrm{~m}$ concentrations that approach the solubility limit for some of them. Alkali halide solutions represent an ideal playground as they range from structure-making (that enhance water structure and retard the dynamics) to structure-breaking (that destroy water structure and accelerate dynamics) cations and anions, ${ }^{1}$ and thus lead to a broad spectrum of acceleration/retardation factors. ${ }^{1,2}$ We first show that available experimental measurements suggest that in general, water reorientation dynamics is not strictly correlated with viscosity. We then use MD simulations to explain this observation by comparing the effects of ions on the local HB exchange dynamics and on viscosity within the extended jump model framework. A critical limitation of non-polarizable MD approaches is the poor description of water dynamics in aqueous salt solutions. ${ }^{14,15} \mathrm{We}$ note that an explicit description of polarization $^{16}$ was used in some studies ${ }^{7,17}$ of dynamical properties in aqueous ionic solutions. However, this treatment remains computationally demanding, while it does not lead to systematically improved dynamical properties with respect to non-polarizable models. ${ }^{14}$

Here, we develop an improved non-polarizable forcefield by implicitly accounting for electronic polarizability via the ECCR ion charge scaling approach. ${ }^{18-22}$ With this new forcefield, simulations are successful in reproducing the NMR measurements of water reorientational and translational dynamics. We also show that the EJM is in excellent agreement with the simulation results for the reorientation dy- 
namics, which allows for a quantitative analysis of the molecular origin for water acceleration/slowdown in these model ionic solutions. Our results unambiguously show that ion-induced changes in water dynamics are usually mostly due to an effect on hydrogen-bond exchange dynamics; the change in viscosity leads to a smaller effect, which governs the retardation only for a minority of salts and at high concentrations.

\section{Methods}

\section{Simulation details}

Molecular dynamics simulations were performed using LAMMPS ${ }^{23}$ (August 2017 serial version). All simulations employed the $\mathrm{SPC} / \mathrm{E}$ water model, ${ }^{24}$ and ion ECCR forcefields ${ }^{18-22}$ partly developed in this work (see below). Long-range electrostatics were treated using Ewald summation, while a short-range real space cut-off of $10 \AA$ for was employed for Lennard-Jones and electrostatics interactions. The water molecule geometry was maintained rigid using the SHAKE algorithm. ${ }^{25}$ We have considered 8 different alkali halide salts at a series of molalities ranging from 1 to $5 \mathrm{~mol} / \mathrm{kg}$. An initial geometry was first generated for each system with PACKMOL. ${ }^{26}$ The box volume was determined so that the density matched the value extrapolated at $300 \mathrm{~K}$ and at the chosen concentration from available experimental data. ${ }^{27}$ Each cubic box consisted of $\approx 1,000$ 1,100 water molecules and between 0 and 90 cations and anions, resulting in boxes of $\approx 31$ $33-\AA$ in side. A first equilibrium simulation was performed in the NVT ensemble $(T=300 \mathrm{~K})$ for 2 ns, using a timestep of 2 fs. 5 configurations were extracted every 200 ps of the last 1 ns of the equilibration. Each of them was then propagated in the micro canonical ensemble for a production run of $100 \mathrm{ps}$, using a timestep of 1 fs. All data was obtained by averaging over these 5 production runs, for a total of 500 ps in each case.

\section{Ion forcefields}

Traditional non-polarizable forcefields are known to provide a poor description of water dynamics in aqueous ionic solutions. In particular, they tend to overestimate the slowdown induced by ions, and fail to reproduce the acceleration observed for some salts, as reported in prior works ${ }^{14,15,28}$ and found in our own exploratory simulations with these models. The importance of polarizability was stressed in ref., ${ }^{15}$ and here we adopt an implicit description of electronic polarizability via the $\mathrm{ECCR}^{18-22}$ ion charge scaling approach. The key aspect of ECCR forcefields is that the charge of all ions is rescaled by a 0.75 factor to implicitly account for the fast electronic contribution to the dielectric constant of water. ${ }^{18}$ In principle these forcefields could be simply obtained by scaling the ion charges in existing ion models. However, the decrease in ionic charge changes the balance between electrostatic and LennardJones interactions and shifts all radial distribution functions (rdf) between oppositely charged atoms to slightly larger distances. Here we followed the strategy suggested by the Jungwirth group ${ }^{20-22}$ and we slightly rescaled the LennardJones radius $\sigma$ in order to recover the correct position for the first peak of the ion-water rdf.

While ECCR forcefields had been obtained in this way for $\mathrm{Li}^{+},{ }^{21} \mathrm{Na}^{+},{ }^{22} \mathrm{~K}^{+19}$ and $\mathrm{Cl}^{-},{ }^{21}$ parameters for the other halide ions were not available. We have therefore selected as a starting point a set of non-polarizable ion forcefield parameters ${ }^{29}$ which have been widely used and successfully describe the structure and thermodynamics of aqueous ionic solutions, but not their dynamics. We have then rescaled the ion charges by 0.75 , and re-optimized the ionic van der Waals radius to conserve the agreement with experimental structural data. ${ }^{30}$ This was achieved by performing several sets of simulations for $1 \mathrm{~m}$ potassium halide solutions, scanning the $\sigma$ value by small decrements until a good agreement with the experimental values ${ }^{30}$ for the position of the first peak of the ion-water oxygen rdf was found. For $\mathrm{F}^{-}: 2.58 \AA$ (experiment), $2.82 \AA$ (no rescaling of $\sigma), 2.58 \AA$ ( $\sigma$ rescaled by $13 \%) ; \mathrm{Br}^{-}$: 
Table 1: Ion parameters employed in the simulations. In cases where we developed the ECCR forcefield $\left(\mathrm{F}^{-}, \mathrm{Br}^{-}\right.$and $\left.\mathrm{I}^{-}\right)$ starting from a full charge model, ${ }^{29}$ the original $\sigma$ value is given between parentheses below the rescaled radius. For the other ions, these parameters are those developed by the Jungwirth group. ${ }^{20-22}$

\begin{tabular}{lrrr}
\hline Ion & Charge $(\mathrm{e})$ & $\sigma(\AA)$ & $\epsilon(\mathrm{kcal} / \mathrm{mol})$ \\
\hline $\mathrm{Li}^{+}$ & +0.75 & 1.800 & 0.01828 \\
$\mathrm{Na}^{+}$ & +0.75 & 2.115 & 0.13009 \\
$\mathrm{~K}^{+}$ & +0.75 & 3.154 & 0.10007 \\
$\mathrm{~F}^{-}$ & -0.75 & 2.984 & 0.10994 \\
& & $(3.43)$ & \\
$\mathrm{Cl}^{-}$ & -0.75 & 4.100 & 0.11778 \\
$\mathrm{Br}^{-}$ & -0.75 & 4.733 & 0.05019 \\
& & $(4.83)$ & \\
$\mathrm{I}^{-}$ & -0.75 & 5.330 & 0.03824 \\
& & $(5.33)$ & \\
\hline
\end{tabular}

$3.37 \AA$ (experiment), $3.43 \AA$ (no rescaling of $\sigma), 3.37 \AA$ ( $\sigma$ rescaled by $2 \%) ; \mathrm{I}^{-}: 3.68 \AA$ (experiment), $3.65 \AA$ (no rescaling of $\sigma$ was necessary). All employed parameters are given in Table 1. Cross-interactions were obtained with the Lorentz-Berthelot mixing rules.

We note that our strategy is conceptually similar to that employed recently in ref. ${ }^{15}$ for two different salts and several water models, but two specific aspects differ. First, the ionic charge rescaling factor employed in ref. ${ }^{15}$ was determined based on the ratio between the experimental and the simulated dielectric constants for a given water model. For the SPC/E model used here, this would lead to a scaling factor of 0.94 , while we used the typical ECCR 0.75 scaling factor. Second, other sets of Lennard-Jones parameters were employed and compared for the ions. Overall, enhanced diffusion was indeed observed for solutions of a structure-breaking salt (CsI), but not with the $\mathrm{SPC} / \mathrm{E}$ water model that we employ here and for which we find that certain combinations of alkali halide ions lead to water dynamics faster than in the bulk.

\section{Analysis}

In each ionic aqueous solution, the average water reorientation time is calculated as the time integral

$$
\left\langle\tau_{\mathrm{r}}\right\rangle=\int_{0}^{\infty} \mathrm{d} t C_{2}(t)
$$

of the reorientation time-correlation function

$$
C_{2}(t)=\left\langle P_{2}[\vec{u}(0) \cdot \vec{u}(t)]\right\rangle,
$$

where $\vec{u}$ is the $\mathrm{OH}$ direction and $P_{2}$ is the second-order Legendre polynomial, and the average is done over all water $\mathrm{OH}$ groups in solution. The frame reorientation time is defined as the reorientation time between successive $\mathrm{HB}$ exchanges. It is calculated in the same way as the full average reorientation time, except that only time intervals between successive HB exchanges are considered. (We note that the frame reorientation time thus depends only on the HB donor-acceptor pair, and not on the future HB acceptor, as incorrectly suggested in some studies. ${ }^{12}$ )

Regarding water translational dynamics, the average translational diffusion coefficient of water is determined by fitting the water oxygen mean-square displacement on the 1-100 ps time interval. As we exclusively focus on the ratios between the diffusion coefficient in a given salt solution and in the bulk, we do not apply finitesize corrections whose effects on the predicted retardation or acceleration are expected to be very small (all simulation boxes being of similar sizes).

The HB jump exchange time $\tau_{\text {jump }}$ is defined as the average time for a water $\mathrm{OH}$ group to go from a stable initial HB acceptor to a new stable $\mathrm{HB}$ acceptor. It is calculated via the cross timecorrelation function between the probabilities to be in the initial (I) and final (F) HB states as

$$
\left\langle p_{I}(0) p_{F}(t)\right\rangle=1-\exp \left(-t / \tau_{\text {jump }}\right)
$$

where $p_{I, F}(t)$ is 1 if the system is in state $\mathrm{I}(\mathrm{F}$ respectively) at time $t$, and 0 otherwise. Following previous work, ${ }^{3}$ stable $\mathrm{HB}$ states defined by tight geometric $\mathrm{HB}$ conditions $\left(R_{\mathrm{OO}}<3.0 \AA\right.$, $\left.R_{\mathrm{HO}}<2.0 \AA, \widehat{\mathrm{HOO}}<21.5^{\circ}\right)$ are used for states 
$\mathrm{I}$ and $\mathrm{F}$ to remove the contributions from fast barrier recrossing. ${ }^{31}$ The values of the cut-off distances are adapted for the different anions: $R_{\mathrm{OF}}<2.85 \AA, R_{\mathrm{HF}}<1.85 \AA ; R_{\mathrm{OCl}}<3.45 \AA$, $R_{\mathrm{HCl}}<2.45 \AA ; R_{\mathrm{OBr}}<3.70 \AA, R_{\mathrm{HBr}}<2.70 \AA$; and $R_{\mathrm{OI}}<4.10 \AA, R_{\mathrm{HI}}<3.10 \AA$. Absorbing boundary conditions in the product state ensure that the forward rate constant is calculated. Previous studies ${ }^{5,7}$ had suggested that the average jump angle $\Delta \theta$ varies very little $\left( \pm 5^{\circ}\right)$ among the halide series, and for simplicity, we assume a constant $\Delta \theta=68^{\circ} .{ }^{5,7}$ The induced error on the jump contribution to the overall reorientation is expected to be $\approx \pm 10 \%$, and that on the total reorientation time estimated from the jump model $\approx \pm 5 \%$. As shown below, the very good agreement between the jump model predictions and the direct calculation of the reorientation times in the simulation suggests that this approximation does not significantly affect our results.

The populations of $\mathrm{OH}$ groups respectively HBed to water molecules and to anions are determined using cut-offs similar to these used for the stable HB, except that all distances are increased by $0.5 \AA$ and the angular condition is $\widehat{\mathrm{HOO}}<30^{\circ}$.

\section{Results and Discussion}

\section{Experimental measurements of wa- ter dynamics}

The dynamics of water in ionic aqueous solutions has been measured with a variety of experimental techniques. ${ }^{3}$ Some of them probe the dynamics of local, molecular motions, including e.g. ultrafast infrared spectroscopy ${ }^{32}$ which can measure the reorientation of water $\mathrm{OH}$ groups, and nuclear magnetic relaxation $(\mathrm{NMR})^{2,33}$ which reports on the reorientation time of different water molecular axes and tensors. Other techniques like terahertz ultrafast spectroscopy ${ }^{34,35}$ and optical Kerr-effect ${ }^{36}$ probe more collective motions, which involve several water molecules. Finally, viscosity is another collective dynamical property which has been extensively measured for a broad range of aqueous ionic solutions. ${ }^{37}$

While all these techniques probe the dynamics of water, they can sometimes lead to seemingly contradictory results regarding the change induced by a given salt on the dynamics of water relative to the bulk. One technique may find an acceleration while another measures a slowdown. Here, we illustrate this point by comparing the changes in viscosity and in the water reorientation time measured by NMR. At a given ion concentration $c$, one considers the water reorientation retardation factor with respect to the bulk

$$
\rho_{r}(c)=\frac{\left\langle\tau_{r}(c)\right\rangle}{\left\langle\tau_{r}(0)\right\rangle}
$$

where $\left\langle\tau_{r}(0)\right\rangle=\tau_{r}^{\text {bulk }}$ is the reorientation time in neat water. $\rho_{r}$ can be indirectly measured by $\mathrm{NMR}^{2,33}$ for the reorientation of the water $\mathrm{HH}$ direction. Similarly, one considers the change in the solution viscosity $\eta$

$$
\rho_{\eta}(c)=\frac{\eta(c)}{\eta^{\mathrm{bulk}}} .
$$

Figure 1 shows how $\rho_{r}$ and $\rho_{\eta}$ evolve for a selection of different alkali halide solutions of increasing concentration. For some salts, the viscosity and NMR-measured water reorientation time exhibit similar changes with respect to the bulk (curves close to the diagonal $\rho_{r}=\rho_{\eta}$ ), yielding both either a slowdown (e.g. KF and $\mathrm{LiCl}$ ) or an acceleration (e.g. KI). However, surprisingly, for some other salts, the viscosity increases while water reorientation becomes faster (e.g. $\mathrm{NaI}$ and $\mathrm{KCl}$ ). Viscosity and water reorientation are thus not systematically affected by ions in the same way.

In order to analyze the effect of ions on viscosity and water reorientation time, and before turning to their concentration-dependence, it is useful to consider the influence of dilute ions. In the dilute regime, the $\rho_{r}$ retardation factor measured by NMR is usually described ${ }^{1,2}$ as a linear function of the ion concentration $c$,

$$
\rho_{r}(c)=1+B_{r} c+o\left(c^{2}\right) .
$$

This led to a classification of individual ions as 


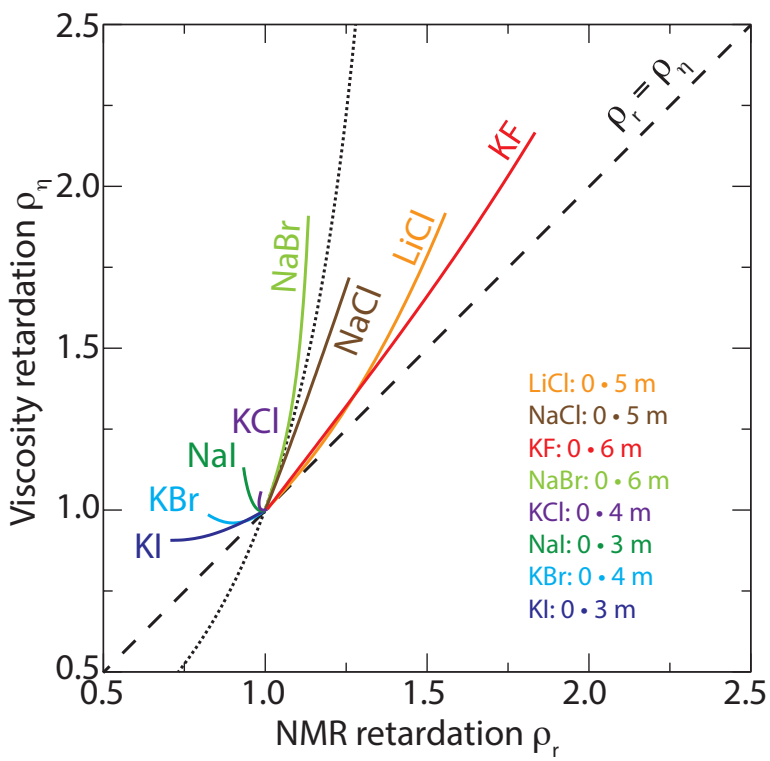

Figure 1: Correlation between changes in experimental viscosity and water reorientation dynamics in alkali halides aqueous solutions (data adapted from Refs. $^{2}$ and ${ }^{38}$ ). The relative scaling of viscosity ${ }^{38} \rho_{\eta}$ is plotted against the scaling of the NMR reorientation time ${ }^{2} \rho_{r}$ for increasing salt concentrations. The dashed line along the diagonal corresponds to the same scaling of both quantities with concentration, i.e. $\rho_{r}=\rho_{\eta}$, while the dotted line corresponds to the putative case where the change in NMR reorientation time would be exclusively due to the change in viscosity (see main text).

structure-maker $\left(\mathrm{B}_{r}>0\right)$ or structure-breaker $\left(\mathrm{B}_{r}<0\right)$ by assuming that the effects of the cation and of the anion were additive, and by performing measurements varying one or the other. $^{1}$

Similarly, changes in viscosity are described in dilute solutions by the Jones-Dole equation ${ }^{1}$

$$
\rho_{\eta}(c)=1+A_{\eta} c^{1 / 2}+B_{\eta} c+o\left(c^{2}\right) .
$$

In general, ${ }^{1}$ the $\mathrm{B}_{\eta}$ terms, which describe the contribution of ion-water interactions to viscosity are found to be close to the $\mathrm{NMR} \mathrm{B}_{r}$ values, which measure the effect of ions on water reorientation. However, Eq. 7 also includes the $A_{\eta}$ term which describes the contribution of ionion interactions to the viscosity change. $A_{\eta}$ is always positive and for alkali halides, it is on the order of $5 \cdot 10^{-3} \mathrm{~L}^{3 / 2} \mathrm{~mol}^{-1 / 2}$ and shows limited variations among the ion series. ${ }^{37}$ In contrast, $B_{\eta}$ is much more ion-specific. ${ }^{37}$ For example, at ambient temperature and for a concentration of $1 \mathrm{~m}$, its contribution ranges from -0.07 for $\mathrm{I}^{-}$ to +0.15 for $\mathrm{Li}^{+} .{ }^{37}$ For a given salt, $B_{r}$ and $B_{\eta}$ are the sum of the individual cation and anion contributions, $B_{r, \eta}=B_{r, \eta}^{+}+B_{r, \eta}^{-}$.

We first examine the dilute regime and study how $\rho_{r}$ and $\rho_{\eta}$ depart from 1 . In most cases in the moderately dilute regime $(0.1-1 \mathrm{~m}),\left|B_{\eta}\right|$ largely exceeds $A_{\eta} / c^{1 / 2} \cdot{ }^{37}$ The good correlation between $\rho_{r}$ and $\rho_{\eta}$ at very low concentration is related to the similarities between $B_{\eta}$ and $B_{r}$ for many salts (dashed line in Fig. 1). In some other cases, such as $\mathrm{NaI}, \mathrm{NaBr}$ or $\mathrm{NaCl}$, the data reported in Fig. 1 suggest that viscosity and reorientation are not perfectly correlated, even in the dilute regime. There are two origins for this phenomenon. First, for some ions, such as $\mathrm{Na}^{+}, B_{\eta}$ can noticeably differ from $B_{r}$, which leads to distinct concentrationdependences. Second, for certains combinations of ions, $B_{\eta}$ becomes comparable, or even dominated, by $A_{\eta} / c^{1 / 2}$, which is always positive. ${ }^{37}$ Examples include combinations of two ions that both weakly perturb dynamics and viscosity $\left(B_{r, \eta}^{+} \simeq 0\right.$ and $\left.B_{r, \eta}^{-} \simeq 0\right)$, such as $\mathrm{KCl}$, or for a structure maker ion neutralizing the effect of a structure breaker counterion $\left(B_{r, \eta}^{+,-} \simeq-B_{r, \eta}^{-,+}\right)$, e.g. $\mathrm{NaBr}$ or NaI, $B_{\eta} \approx B_{r} \approx 0$. However, while the reorientation dynamics is weakly perturbed, viscosity increases because of the $A_{\eta} c^{1 / 2}>0$ contribution, leading to a decorrelation between the changes in viscosity and reorientation dynamics.

Now turning to higher concentrations, the experimental data of Fig. 1 suggest that for all the salts, the viscosity change $\rho_{\eta}$ becomes increasingly more pronounced than $\rho_{r}$ as concentration increases. In particular, a linear relationship between $\rho_{\eta}$ and $\rho_{r}$ is never observed at concentrations higher than $\approx 1 \mathrm{~m}$. Strikingly, for strong structure-breakers such as $\mathrm{KBr}$ or NaI, water reorientation dynamics is always accelerated at all investigated concentrations while viscosity always increases at large concentration. For KI, which leads to the most pronounced accelerations of water dynamics, viscosity is even 
decreased on the $0-3 \mathrm{~m}$ concentration range, but less so than reorientation dynamics.

Therefore, experimental results demonstrate that the effects of ions on viscosity and on water reorientation dynamics are not always similar and show a markedly distinct concentrationdependence.

This has important consequences for the molecular mechanism of water reorientation in aqueous salt solutions. As described in the introduction, two independent processes contribute to water reorientation: the HB exchange dynamics and the tumbling of the intact HB between exchange events. ${ }^{8}$ Only the second term is approximately diffusive, ${ }^{8}$ and thus as we showed in a prior study, ${ }^{7}$ it scales with the solution viscosity. ${ }^{12}$ By anticipating the detailed analysis that will be presented further, one can actually use the experimental measurements to infer the contribution of the increase/decrease in viscosity to the NMR reorientation time. The dotted line in Fig. 1 represents the expected NMR retardation if only the tumbling component was affected, and the HB exchange dynamics left unperturbed by the ions as compared to the bulk. In most cases, the experimental curves strongly deviate from this behavior, suggesting that in these solutions the viscosity increase is not the main cause for the retarded dynamics, while the opposite is true for two salts $(\mathrm{NaCl}$ and $\mathrm{NaBr})$. These experimental results therefore strongly differ from the conclusions of a previous study on sodium and potassium thiocyanate. ${ }^{12}$ It was suggested that the increase in viscosity was the main reason for retarded dynamics at high concentrations, a conclusion that was meant to be general for all ion solutions. ${ }^{12}$ In contrast, the above-described experimental results support the conclusions of our prior study, ${ }^{7}$ where we suggested that the increased viscosity becomes important at high concentration for some specific salts such as sodium perchlorate, but that this does not apply to many other salts where viscosity has a minor effect on water dynamics.

\section{MD simulations with ECCR force- fields}

In order to provide a detailed molecular interpretation that could rationalize these experimental observations, we have performed atomistic molecular dynamics simulations of the corresponding solutions (see Methods). Previous studies have shown that typical non-polarizable ion forcefields fail to reproduce the accelerated dynamics observed for some salts, ${ }^{14,15,39,40}$ as confirmed in the present study by additional exploratory simulations of salt solutions at various concentrations.

To resolve this issue, we have tested the recent suggestion to multiply the charge of all ions by 0.75 , in order to implicitly account for the fast electronic contribution to the dielectric constant of water ${ }^{18}$ (ECC approach, see Methods). Additional corrections to the fullcharge models were made to the ion radius in order to maintain the correct hydration structure when the charge is reduced, hereafter denoted the ECC-rescaled (ECCR) approach. ${ }^{18-22}$ Parameters were available for the alkali cations and $\mathrm{Cl}^{-},{ }^{19-22}$ and we adapted some widely used parameters for the other halides ${ }^{29}$ in order to obtain the corresponding ECCR forcefields (Table 1). Such an ECCR approach was recently shown to successfully reproduce water translational dynamics for a selection of alkali halide salts. ${ }^{15,28}$

We now assess the ability of this new set of ECCR forcefields to describe the effect of ions on the rotational dynamics of water. We focus on the reorientation of water $\mathrm{OH}$ groups, because this is the vector that reorients during HB jump exchanges and that will be described with the jump model in the next section. However, experimental proton NMR measurements report on the rotational relaxation of another axis, the $\mathrm{HH}$ vector. ${ }^{33}$ While water reorientation is not strictly isotropic, especially in the presence of ions, very little decoupling was observed between the reorientation of various molecular axes next to halide anions. ${ }^{5}$ We thus compare the slowdown of the (simulated) $\mathrm{OH}$ bond reorientation time and the experimentally-determined value for the $\mathrm{HH}$ 


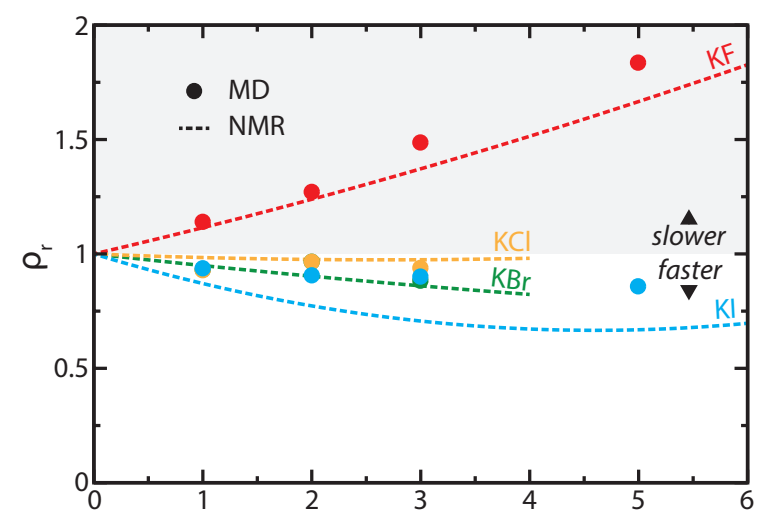

A

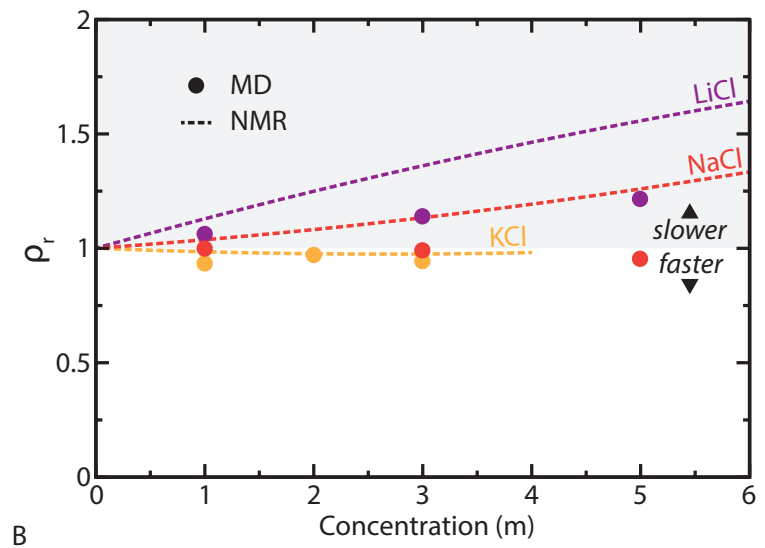

Figure 2: Comparison between NMR and MD simulations results for water reorientation dynamics for alkali halide salts at increasing concentrations. The ratio $\rho_{r}$ is shown for $(\mathrm{A})$ solutions of potassium halides and (B) alkali chlorides.

axis. We stress that this slowdown calculation is averaged over all water $\mathrm{OH}$ groups in the solution, and includes water molecules in different environments, ranging from a bulk-like environment to a direct contact with an ion.

In Fig. 2, we show the $\rho_{r}$ values (Eqs. 1, 2 and 6) from our ECCR simulations and from experimental NMR measurements. While the agreement with experiments is not always quantitative, the simulation results exhibit all the key features of these solutions. First, water dynamics is accelerated for strong structurebreaking salts such as KI or KBr, while this was not correctly reproduced by prior force fields. ${ }^{14}$ Second, a good agreement with experimental data is observed for the potassium halide series (Fig. 2A). In particular, at all concentrations, dynamics slows down when going from $\mathrm{I}^{-}$to
$\mathrm{F}^{-}$. Simulations are quantitative for $\mathrm{KF}, \mathrm{KCl}$ and $\mathrm{KBr}$ at all investigated concentrations, and slightly too slow for $\mathrm{KI}$ at $5 \mathrm{~m}$. When varying the cation (Fig. 2B), the trend going from $\mathrm{K}^{+}$ to $\mathrm{Li}^{+}$is correctly reproduced, but the retardation is always underestimated as compared to the experiments for $\mathrm{Na}^{+}$and $\mathrm{Li}^{+}$. In particular, the present ECCR forcefields lead to very small differences between $\mathrm{K}^{+}$and $\mathrm{Na}^{+}$, and do not fully reproduce the very large slowdown induced by $\mathrm{Li}^{+}$. The recent comparison of water reorientation dynamics in $\mathrm{KSCN}$ and $\mathrm{NaSCN}$ solutions ${ }^{12}$ also suffered from a limited difference between the two cations as compared to the experimental results, even if the trends were correctly reproduced.

We now examine the ability of the ECCR forcefields to reproduce the effect of ions on water translational dynamics measured experimentally. ${ }^{41}$ We consider the ratio

$$
\frac{1}{\rho_{\text {trans }}}=\frac{D(c)}{D^{\text {bulk }}},
$$

where $D(c)$ and $D^{\text {bulk }}$ are the water translational diffusion coefficients in the presence of salt and in the bulk. The values of $1 / \rho_{\text {trans }}$ from our simulations and from experimental data are shown in Fig. 3A and C. For the halide series (Fig. 3A), the experimental trend is very well reproduced, and accelerated diffusion is indeed measured for $\mathrm{KBr}$ and $\mathrm{KI}$ for low concentrations, while it is very much slowed down in KF solutions, for which the agreement with experimental data is fully quantitative. However, in $3 \mathrm{~m}$ and $5 \mathrm{~m} \mathrm{KI}$ solutions, translational dynamics is slowed down in the simulations whereas in the experiments it remains faster than in the bulk. When varying the cation in alkali chloride solutions (Fig. 3C), the experimental trend is reproduced. Simulations are in quantitative agreement for $\mathrm{KCl}$ and $\mathrm{LiCl}$ over a wide concentration-range, while dynamics is retarded as compared to the bulk by slightly faster than in the experiments for $\mathrm{NaCl}$, once again suggesting that the current forcefield underestimates the retardation induced by the $\mathrm{Na}^{+}$cation.

Translational diffusion results are in agree- 

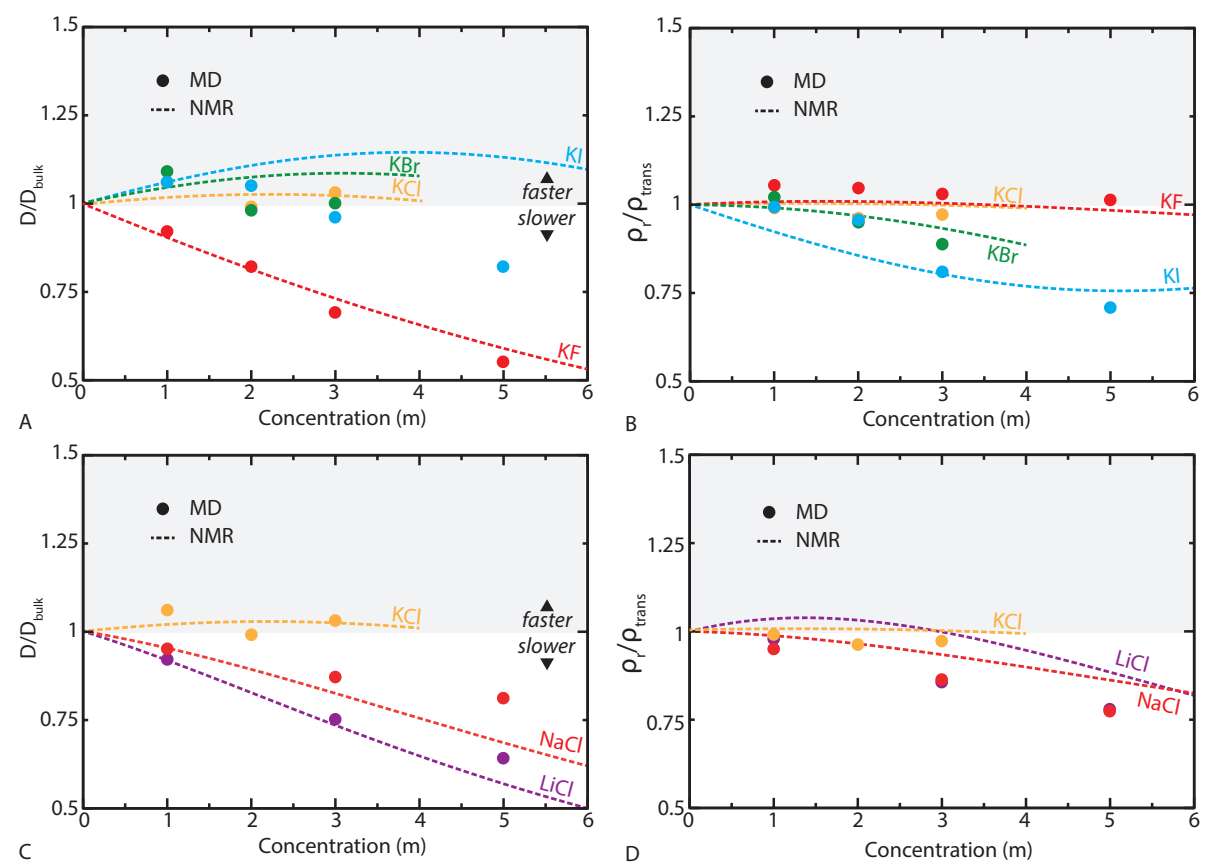

Figure 3: Water translation diffusion coefficients for alkali halide salts at increasing concentrations (experimental data taken from ref. ${ }^{41}$ ). The ratio $1 / \rho_{\text {trans }}$ between the diffusion coefficient of water molecules in the presence of salt and in the bulk is shown for (A) solutions of potassium halides and $(\mathrm{C})$ alkali chlorides. The rotational-translation decoupling, as estimated by the ratio $\rho_{r} / \rho_{\text {trans }}$ is shown for (B) solutions of potassium halides and (D) alkali chlorides.

ment with previous attempts to reproduce enhanced or retarded water diffusion in aqueous salt solutions using scaled-charges models. ${ }^{15,28}$ For $\mathrm{NaCl}$, the current results are close to those of the best-performing model of ref. ${ }^{15}$ (E3B water and MP-S ion-forcefield), slightly underestimating the experimental retardation, while in ref., ${ }^{28}$ an ion model specifically reparameterized together with the TIP4P/2005 water model overestimated the experimental retardation. Nonetheless, all the models that employ rescaled ion charges largely outperform usual full-charges models, which lead to retardations much larger than in the experiments. ${ }^{14,15,28}$

We finally examine the water rotationtranslation decoupling in aqueous salt solutions (Fig. 3B and D). Experimentally, the decoupling is much more pronounced when the anion is varied in potassium halide solutions than when the cation is varied in alkali halide solutions. This behavior is well-captured in our simulations. In particular, at increasing concentrations, rotational dynamics tends to be less retarded than translational dynamics, except for KF where there is almost no decoupling on the entire concentration-range, which is both observed experimentally and in our simulations. The rotational-translation decoupling does not seem to be correlated with viscosity, as the more viscous solutions $(\mathrm{LiCl}, \mathrm{KF})$ do not exhibit the largest decouplings.

Our results therefore demonstrate that ECCR models capture all the essential features of water dynamics in alkali halide solutions: retarded or accelerated dynamics, concentration effects, trends upon changing the cation or the anion, and the rotational-translational decoupling. For several salts, the results are in quantitative agreement with the experimental data. Future improvements will be required to quantitatively reproduce the distinct effects of the different alkali cations on water dynamics, for which the correct trend is obtained but that do not quite show the dramatic differences measured experimentally. However, our ECCR results show that these simulations can be analyzed to elucidate the sometimes contrasted effects of ions on viscosity and NMR reorientation 
times.

\section{Extended-jump model analysis of water reorientation}

We now introduce the extended jump model (EJM) for water reorientation: this model showed that water reorientation arises from two independent processes, with only one of them correlated to the solution viscosity. ${ }^{8}$ We then use our simulations to determine the respective contributions of these two processes for the range of salts and concentrations studied here.

Beyond the fast librational decay $(<0.2 \mathrm{ps})$, the EJM showed that $\mathrm{OH}$ groups reorient via two mechanisms: first, via large amplitude angular jumps where a water $\mathrm{OH}$ group switches hydrogen-bond (HB) acceptors; second, via a diffusive reorientation of the frame formed by an intact HB axis between two successive jumps. ${ }^{3,8}$ In the bulk, on the picosecond timescale the second-order Legendre polynomial $P_{2}$ (Eq. 2) decays exponentially with a time constant $\tau_{2}$ that can thus be expressed as

$$
\begin{aligned}
\tau_{2}^{-1} & =\left(\tau_{\text {reor }}^{\text {jump }}\right)^{-1}+\left(\tau_{\text {raor }}^{\text {frame }}\right)^{-1} \\
& =\tau_{\text {jump }}^{-1}\left[1-\frac{1}{5} \frac{\sin (5 \Delta \theta / 2)}{\sin (\Delta \theta / 2)}\right]+\left(\tau_{\text {reor }}^{\text {frame }}\right)^{-1}(9)
\end{aligned}
$$

where $\tau_{\text {reor }}^{\text {jump,frame }}$ are the jump and frame contributions to the reorientation time, $\tau_{\text {jump }}$ is the jump time (the inverse HB exchange rate), and $\Delta \theta$ is the average jump amplitude. ${ }^{3,8}$ Note that this long-time $\tau_{2}$ slightly differs from the integrated reorientation time $\tau_{r}$ discussed in the previous section (Eq. 1), which incorporates the effects of the fast librational component and is therefore slightly faster.

In aqueous ionic solutions, anions can accept HBs from water molecules. HB exchanges in the solution can thus occur starting from either a water molecule (W) or an anion (A) initial acceptor. When averaging over the entire solution, these two populations with probabilities $p_{W}$ and $p_{A}$ will thus decay with distinct reorientation time constants $\tau_{2}^{W}$ and $\tau_{2}^{A}$, and the average $\left\langle\tau_{2}\right\rangle$ is a linear combination of the two

$$
\left\langle\tau_{2}\right\rangle=p_{W} \tau_{2}^{W}+p_{A} \tau_{2}^{A} .
$$

$\tau_{2}^{W}$ and $\tau_{2}^{A}$ are estimated independently by calculating the jump and frame components in eq. 9 for an $\mathrm{OH}$ initially HBed to a water molecule or to an anion, and their respective (normalized) populations (see Methods). Finally, the ratio between this EJM estimation of the average $\left\langle\tau_{2}\right\rangle$ in each solution and the bulk is compared to the raw simulation results, and to the experiments, as shown in Fig. 4. The retardation/acceleration predicted by the EJM is in remarkable agreement with that calculated directly from the average time constant of the reorientation tcf (Eq. 1). In all cases, the trend observed in the different salts at increasing concentrations is fully captured by the EJM. Fig. 4 also highlights the overall good agreement between the simulations and the NMR experiments as discussed before, with the exception of a few cases (such as $\mathrm{LiCl}, \mathrm{NaCl}$ or $\mathrm{NaBr}$ ), where the agreement is less quantitative even if the trend when varying the cation or the anion is correctly captured. These results validate the EJM decomposition, which had been shown to be successful in a wide range of aqueous solutions, $, 3,5,7,9-12,42,43$ at interfaces, ${ }^{3,44-48}$ in confinement ${ }^{49}$ and in supercooled conditions. ${ }^{50}$

Therefore, we now turn to the interpretation of the distinct effects of the various salts by comparing the contributions of jumps and of the frame reorientation in the presence of the ions.

In the bulk, reorientation via jumps is significantly faster than reorientation via tumbling of the local frame. The jumps $\left(\tau_{\text {reor }}^{\text {jump }}=\right.$ $3.3 \mathrm{ps}$ ) account for approximately two thirds of the $\mathrm{OH}$ group long-time reorientation rate $\left(\tau_{2}^{\mathrm{EJM}}=2.1 \mathrm{ps}\right)$, while the frame reorientation $\left(\tau_{\text {reor }}^{\text {frame }}=5.7 \mathrm{ps}\right)$ brings a more minor contribution. One important result from our analysis is that jumps remain the dominant reorientation pathway in all investigated solutions, including solutions where dynamics is strongly accelerated or retarded. Jumps are typically responsible for $\approx 60-70 \%$ of the water reorientation rate, with the most extreme cases being KF $(55 \%)$ or $\mathrm{LiCl}(74 \%)$ at $5 \mathrm{~m}$. We therefore do not find any example where the frame reorientation would become the dominant reorientation pathway. 

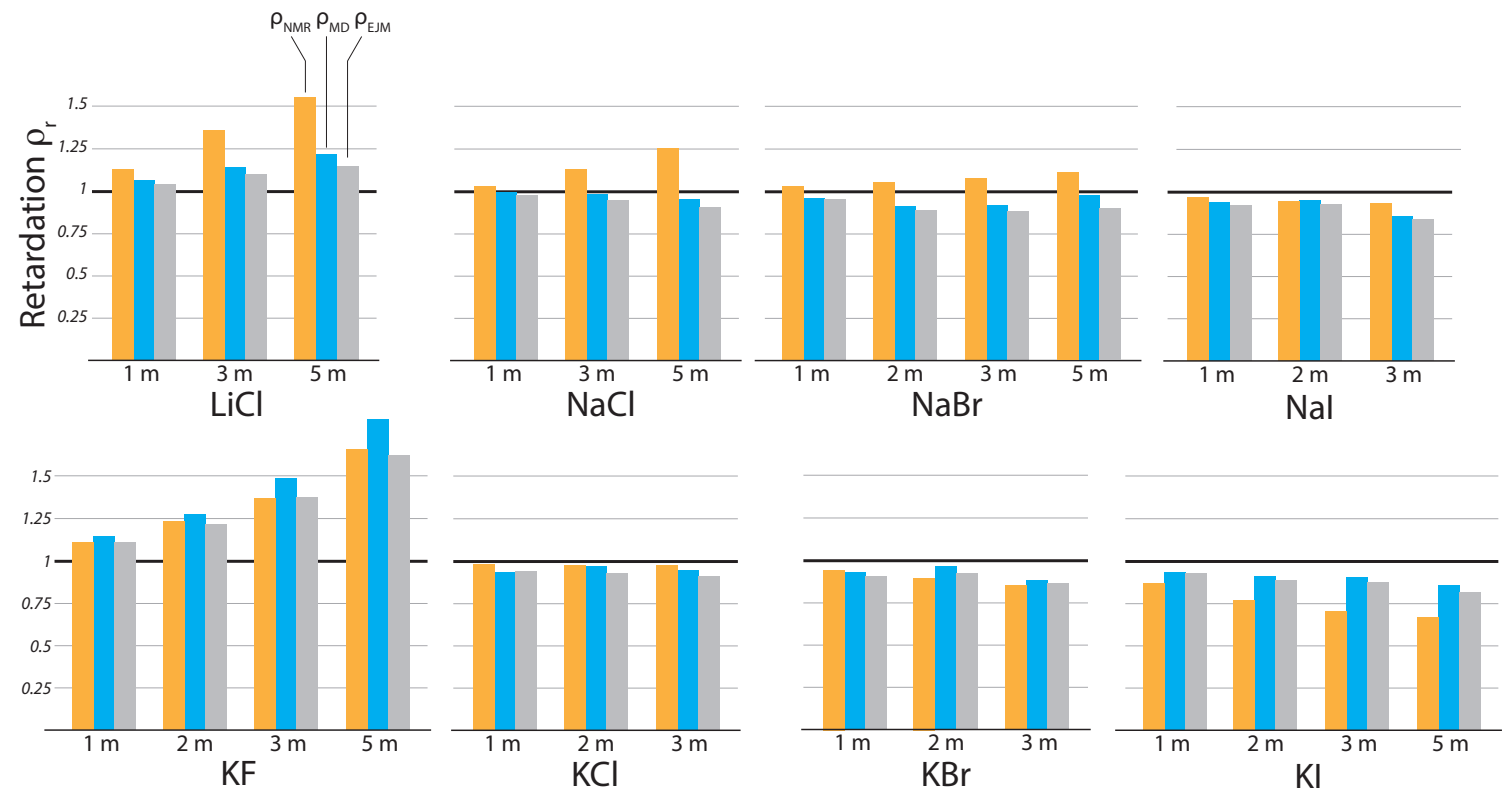

Figure 4: Comparison between the acceleration/retardation of water reorientation dynamics $\rho_{r}$ in alkali halide solutions at different concentrations, as measured in NMR ( $\rho_{\mathrm{NMR}}$, orange), in the simulations using Eqs. 1-2 ( $\rho_{\mathrm{MD}}$, blue), and using the EJM decomposition in Eqs. 9-10 ( $\rho_{\mathrm{EJM}}$, gray).

Ions, and anions in particular, have a dramatic effect on the HB exchange time. While the HB exchange time in the bulk is $\tau_{\text {jump }}=$ $3.1 \mathrm{ps}$, jumps starting from an anion in e.g. 1$\mathrm{m}$ potassium halide solutions are significantly affected, ranging from $\tau_{\text {jump }}=2.2$ ps for $\mathrm{I}^{-}$, $2.4 \mathrm{ps}$ for $\mathrm{Br}^{-}, 3.7 \mathrm{ps}$ for $\mathrm{Cl}^{-}$, to $12.6 \mathrm{ps}$ for $\mathrm{F}^{-}$, demonstrating that there is a factor of $\approx 5$ between the most accelerating and most retarding halides. Indeed, we had shown that the effect of a solute on the HB exchange time can be understood by a combination of two effects. ${ }^{3,9,10}$ First, an excluded-volume effect, where the solute hinders the approach of a new HB acceptor, present for all solutes or interfaces. Second, for HB acceptors, there is an additional contribution corresponding to the free-energy cost to stretch the $\mathrm{HB}$ between the reorienting $\mathrm{OH}$ group and its acceptor. ${ }^{3,10}$ Depending on the strength of this HB as compared to bulk water, this can lead to an acceleration (in the present case, for weak acceptors such as $\mathrm{I}^{-}$) or to a retardation (strong acceptors such as $\mathrm{F}^{-}$).

By contrast, the frame reorientation time exhibits much more limited variations. In the bulk, $\tau_{\text {reor }}^{\text {frame }}=5.7 \mathrm{ps}$, and while it is slightly accelerated at low concentrations of structurebreaking salts such as $\mathrm{KBr}\left(\tau_{\text {reor }}^{\text {frame }}=5.1\right.$ ps at 1 $\mathrm{m})$, it is most often increasingly retarded with concentration, but at most by a factor of 1.8 in $\mathrm{KF}$ at $5 \mathrm{~m}$. In contrast to the effect of ions on HB exchanges is local, variations of the frame reorientation are due to the change in solution viscosity $\eta$. Indeed the frame reorientation is approximately diffusive, ${ }^{8}$ and it can therefore be described by the Debye-Stokes-Einstein equation. The frame retardation $\rho_{\text {frame }}$ should thus scale with $\rho_{\eta}$, as clearly shown in previous studies. $^{7,12}$ We had previously suggested that the ion effects on viscosity could actually be understood within the simple microscopic model developed by Eyring, ${ }^{51}$ where viscosity scales as

$$
\eta \propto \tau_{\mathrm{tj}} / L_{\mathrm{tj}}^{2}
$$

where $1 / \tau_{\mathrm{tj}}$ and $L_{\mathrm{tj}}$ are the rate and amplitude of translational jumps. Since these are induced by HB exchanges, $\tau_{\mathrm{tj}}$ can be equalled to $\tau_{\text {jump }}$ (Eq. 9) and $L_{\mathrm{tj}}$ to the translational displacement induced by a HB exchange event. Even if this description is clearly very approximate for an associated liquid like water ${ }^{51}$ and it neglects the explicit effect of the solutes, it was shown to be remarkably successful up to the concentrated regime for aqueous salt solutions ${ }^{12}(\simeq 5 \mathrm{M})$. Failures were only observed at extreme concen- 
trations (10-15 M), which are likely due to limitations of this simplified description, and do not necessarily affect the applicability of the jump model, in contrast to what was implied in ref. ${ }^{12}$

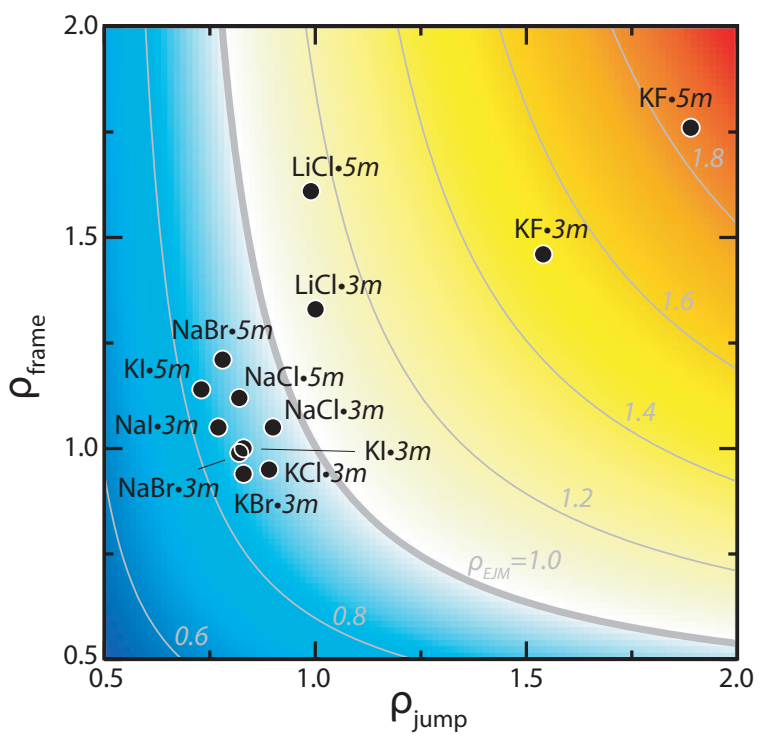

Figure 5: Jump ( $\rho_{\text {jump }}, x$ axis $)$ and frame ( $\rho_{\text {frame }}, y$ axis) average scaling for all solutions at concentrations $\geq 3 \mathrm{~m}$. The color scale indicates the total retardation $\rho_{\mathrm{EJM}}$, with contourlines of constant increment indicated in gray.

We can now use the decompositions of Eq. 9 and Eq. 10 to estimate how the average frame and jump times vary in the salt solutions (calculated using the two-state approach described in Eq. 10), and how they contribute to the overall retardation of $\left\langle\tau_{2}^{\text {EJM }}\right\rangle$ (Fig. 5).

Since jumps are the dominant reorientation pathway in the bulk, any acceleration or retardation of the HB exchange time $\rho_{\text {jump }}$ has a much more pronounced effect than an alteration $\rho_{\text {frame }}$ of the frame contribution. This is clearly evidenced in the representation of Fig. 5, where the overall effect is more sensitive to variations along the horizontal axis $\left(\rho_{\text {jump }}\right)$ as compared to the vertical one ( $\left.\rho_{\text {frame }}\right)$.

The importance of jumps in determining the overall acceleration or slowdown of the dynamics is well-illustrated in e.g. concentrated solutions of $\mathrm{KI}$ or $\mathrm{NaBr}$, where opposite effects are observed for the frame reorientation, which is slower than in the bulk, and the jump contribution, which is faster than in bulk because of the weak $\mathrm{HBs}$ between water and the $\mathrm{Br}^{-}$or the $\mathrm{I}^{-}$ions. The overall reorientation is clearly accelerated, with $\rho_{\mathrm{EJM}} \approx \rho_{\text {jump }}$ (for example, for KI at $5 \mathrm{~m}, \rho_{\text {frame }}=1.14, \rho_{\text {jump }}=0.73$ and $\left.\rho_{\mathrm{EJM}}=0.84\right)$.

In connection with the observed accelerating effect of $\mathrm{I}^{-}$, we pause to discuss a very recent computational study ${ }^{52}$ focusing on the effect of an iodide ion on the dynamics of water in small $\mathrm{I}^{-}\left(\mathrm{H}_{2} \mathrm{O}\right)_{2}$ clusters. Simulations explicitly accounting for electronic and nuclear quantum effects found that $\mathrm{I}^{-}$induces an acceleration of the cluster HB dynamics, which was contrasted with an apparent iodide-induced slowdown measured in ultrafast IR spectroscopy for ambient-temperature high-concentration aqueous solutions. It was concluded that cooperative $\mathrm{HB}$ dynamics beyond the first hydration shell is responsible for the slowdown in solution. However, we stress that extensive NMR measurements have shown ${ }^{2}$ that iodide accelerates water dynamics even in aqueous solutions. The slowdown measured in ultrafast spectroscopy does not directly reflect the impact of a single iodide on water reorientation dynamics, since measurements are done on very concentrated solutions, and it was shown earlier that the anisotropy decay measured by this technique can suffer from a bias caused by different vibrational lifetimes. ${ }^{6}$ Our present work shows that classical MD simulations reproduce the experimental NMR data and that the faster dynamics is mainly due to the weaker water- $\mathrm{I}^{-}$HBs as compared to water-water HBs, which results in faster jump times. This effect is thus fully captured by our simulations and interpreted in the framework of the EJM. We further stress that HB rearrangements in low-temperature clusters involve pathways that are usually little relevant for ambient aqueous solutions. For example, tunneling is important in clusters ${ }^{52}$ while it is not in the liquid at ambient temperature; ${ }^{53}$ moreover, in clusters, many water $\mathrm{OH}$ groups are dangling and can thus easily reorient, while they are HBed in solution. Dangling OH groups can be observed at interfaces, but their population and the local water geometry are very different from that of water clusters.

The importance of jumps is further illustrated in solutions that retard water reorientation, 
such as LiCl. For this salt, jumps are not retarded (at $\left.5 \mathrm{~m}, \rho_{\text {jump }}=1.00\right)$, the frame reorientation is much more affected $\left(\rho_{\text {frame }}=1.61\right)$ but the overall retardation is clearly closer to that of the jumps $\left(\rho_{\mathrm{EJM}}=1.15\right)$. Only in cases where the frame and the jump contributions are affected similarly (either slightly accelerated, such as in $\mathrm{KCl}$ at $3 \mathrm{~m}$, or strongly retarded such as in $\mathrm{KF}$ ), the overall retardation is logically $\rho_{\mathrm{EJM}} \approx \rho_{\text {frame }} \approx \rho_{\text {jump }}$. In all cases, as mentioned before, jumps remain the main reorientation pathway even in cases where the retardation compared to the bulk is mostly due to viscosity.

\section{Experimental perspectives on the jump and frame contributions to water reorientation}

We now show that the molecular understanding provided by the EJM approach can be used to infer the contributions from the changes in the water HB exchange time and in the frame tumbling directly from experiments, thus avoiding any force-field limitation. Such decomposition is important to guide the choice of an electrolyte with controlled dynamics. Two ionic aqueous solutions with similar overall water reorientation times may have very different local water dynamics depending on the jump and frame contributions. Because the frame is affected by viscosity, which is a more global property, while jumps are sensitive to the nature of the local HB partner, solutions where the jump contribution is dominant may exhibit a larger dynamical heterogeneity than solutions with a similar average reorientation time where viscosity dominates. In addition, chemical processes such as proton transfer reactions are governed by $\mathrm{HB}$ exchanges in the first hydration shell. ${ }^{54}$ The approach described below may thus be relevant for example to analyze the dynamical properties of electrolytes in batteries and in aqueous solar cells (We finally note that the jump time can be directly accessible experimentally via twodimensional infrared spectroscopy ${ }^{55-57}$ for anions causing a large $\mathrm{OH}$ stretch vibrational frequency shift).
In this approach, the only ingredients obtained from the MD simulations are the bulk reorientation components, $\tau_{\text {reor }}^{\text {jump }}=3.3 \mathrm{ps}$ and $\tau_{\text {reor }}^{\text {frame }}=5.7 \mathrm{ps}$ (leading to $\tau_{2}^{\mathrm{EJM}}=2.1 \mathrm{ps}$ ). In the presence of salts, experimental measurements allow to estimate the scaling of the overall reorientation time (NMR data) and that of the frame tumbling (viscosity data). The scaling of the jump time is then estimated using Eq. 9. Finally, for each salt and at each concentration $c$, the difference

$$
\Delta \tau(c)=\tau_{2}^{\mathrm{EJM}}(c)-\left.\tau_{2}^{\mathrm{EJM}}\right|_{\text {bulk }}
$$

can be approximated in a perturbative approach as the sum of the similar differences defined for the jump (HB) and frame (viscosity) components:

$$
\Delta \tau(c) \approx \Delta \tau_{\mathrm{HB}}(c)+\Delta \tau_{\eta}(c)
$$

that are estimated from the experimental measurements as described above. Results are shown in Fig. 6 for the eight salts at concentrations where both concentration-dependent NMR reorientation time ${ }^{2}$ and viscosity data were available. ${ }^{38}$ Since the reorientation time measured by the NMR experiments is that of the $\mathrm{HH}$ vector, whereas the EJM decomposition is originally derived for the $\mathrm{OH}$ vector, we again make the approximation that water reorientation is almost isotropic, which was shown to be reasonable next to halide anions. ${ }^{5}$

Our MD simulations have shown the importance of the jumps in determining the overall acceleration or slowdown of the dynamics. Similarly, for most salts (6 out of 8$), \Delta \tau_{\mathrm{HB}}$ exceeds $\Delta \tau_{\eta}$ at all investigated concentrations. In particular, the faster reorientation dynamics observed in e.g. $\mathrm{KBr}$, KI, or $\mathrm{NaI}$, is nonambiguously due to a major accelerating contribution of the HB exchange dynamics, leading to $\Delta \tau_{\mathrm{HB}}<0$. For these salts, the viscosity contribution to the overall acceleration is negligible, and for NaI, of opposite sign. For strong retardants ( $\mathrm{KF}$ or $\mathrm{LiCl}$ ), both an increased viscosity and slower HB dynamics contribute to the overall retardation, but the jump contribution remains slightly dominant. 


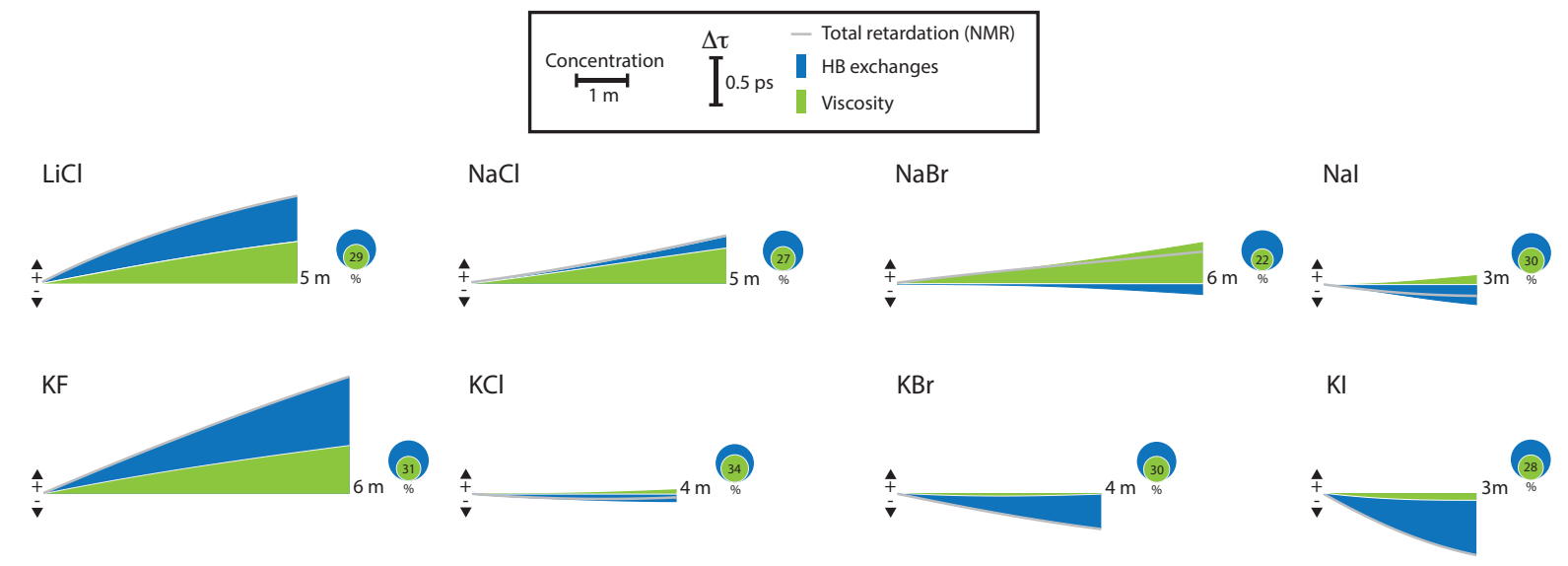

Figure 6: Viscosity (green) and jump HB exchange (blue) contributions (Eq. 13) to the difference between overall reorientation time in each solution and in the bulk estimated using the EJM interpretation of experimental viscosity and NMR measurements. The green and blue disks indicate the absolute contributions of the HB exchange and of the viscosity components in Eq. 9 at the highest investigated concentration.

As anticipated in the discussion of Fig. 1, in two cases $(\mathrm{NaCl}$ and $\mathrm{NaBr})\left|\Delta \tau_{\eta}\right|>\left|\Delta \tau_{\mathrm{HB}}\right|$, and the overall retardation is thus mainly due to the viscosity increase. These are salts for which the increase in viscosity is much more pronounced than the retardation of the NMR reorientation time, pointing to an overall mild effect of the salt on the HB exchange dynamics (slightly retarded in $\mathrm{NaCl}$ and accelerated in $\mathrm{NaBr}$ ). However, we stress that in both solutions, as well as in all the other ones, water reorientation still mainly proceeds by $\mathrm{HB}$ exchange, even at the highest investigated concentrations (blue and green disks in Fig. 6), accounting for approximately the two-thirds of the reorientation. We finally note that since $\tau$ is not directly the sum of $\tau_{H B}$ and $\tau_{\eta}$, the perturbative decomposition of Eq. 13 leads to deviations from the experimentally measured $\Delta \tau_{\mathrm{NMR}}$ when the retardation/acceleration effects are large. In the present cases, noticeable deviations are only observed between $\mathrm{LiCl}$ and $\mathrm{KF}$ at the highest investigated concentrations, for which the total $\Delta \tau_{\mathrm{NMR}}$ is underestimated by $\approx 20-30 \%$.

The experimental data therefore suggest that multiple scenarios exist. This is in contrast with a recent study ${ }^{12}$ which had suggested that results obtained for two salts would be general and that slowed water dynamics in all ionic aqueous solutions is caused by a viscosity effect. Our present results show that while this is the case for some specific salts and while viscosity can never be ignored, jumps are the dominant factor for most solutions.

We now use the representation of Fig. 1 to directly identify regions where jumps (resp. viscosity) are dominant in the ion-induced effect on water dynamics. In the plot along $\rho_{\eta}$ and $\rho_{r}$ in Fig. 1, we have highlighted the regions where the viscosity component dominates the overall effect on the NMR reorientation time $\left(\left|\Delta \tau_{\eta}\right|>\left|\Delta \tau_{\mathrm{HB}}\right|\right.$, gray zone in Fig. 7A). As expected, there are only 2 alkali halide salts for which the NMR retardation at high concentration is in fact mainly due to an increased viscosity. For all the other salts, the HB contribution dominates.

While the present analysis has been so far applied to the alkali halide solutions, we now extend it to other salts. In Fig. 7B, we report the same plot along $\rho_{\eta}$ and $\rho_{r}$ in various salt solutions containing molecular anions and for which the NMR reorientation time was reported in Ref. ${ }^{2}$ Because the concentration-dependent viscosity was not available for all these solutions, we used the $B_{r}$ and $B_{\eta}$ to estimate the concentration-dependence of the NMR reorientation time and of viscosity (Eqs. 6 and 7; the $A_{\eta}$ term was neglected as it is often negligible 
outside of extreme dilution). As these equations are only valid in the dilute regime in principle, the data in Fig. 7B are only extrapolated up to $3 \mathrm{~m}$. When the concentration-dependent viscosity data was available at a higher concentration, the corresponding data point was included in order to estimate the limits of the linear approach.

The results show that the different scenarios observed for alkali halides are also found for other types of salts. For most of them, the HB exchange dynamics brings the dominant contribution to the observed scaling of the NMR reorientation time, while the viscosity effect is dominant for only a few of them. However, the limits of the current approach that use estimations of the NMR reorientation time and of viscosity based on the $B$ factors is clearly evident from the strong deviations from the linear behavior observed in a few selected cases, including $\mathrm{NaClO}_{4}$ that enters the zone where $\left|\Delta \tau_{\eta}\right|>\left|\Delta \tau_{\mathrm{HB}}\right|$ at $4 \mathrm{~m}$, as correctly reproduced and rationalized in earlier simulation work. ${ }^{7}$ Similar deviations are expected for $\mathrm{NaSCN}$, for which simulations indeed suggested that the overall retardation was dominated by changes in viscosity. ${ }^{12}$

\section{Concluding Remarks}

We have studied water dynamics in aqueous solutions of alkali halide from dilute concentrations up to the concentrated regime. Experimental measurements suggest that ions can have a wide range of impacts on the water translational and reorientational dynamics, depending on the nature of the ions and on their concentration. ${ }^{1,2}$ While viscosity is often suggested to be well correlated with the retardation measured by NMR on reorientation dynamics in the dilute regime, ${ }^{1}$ we show here that this is not necessarily the case. At large concentrations, viscosity is always increased ${ }^{38}$ relative to the bulk and more affected than the water reorientation dynamics. For several salt solutions, viscosity and reorientation dynamics change in opposite directions, with the reorientation dynamics being accelerated while the viscosity in-
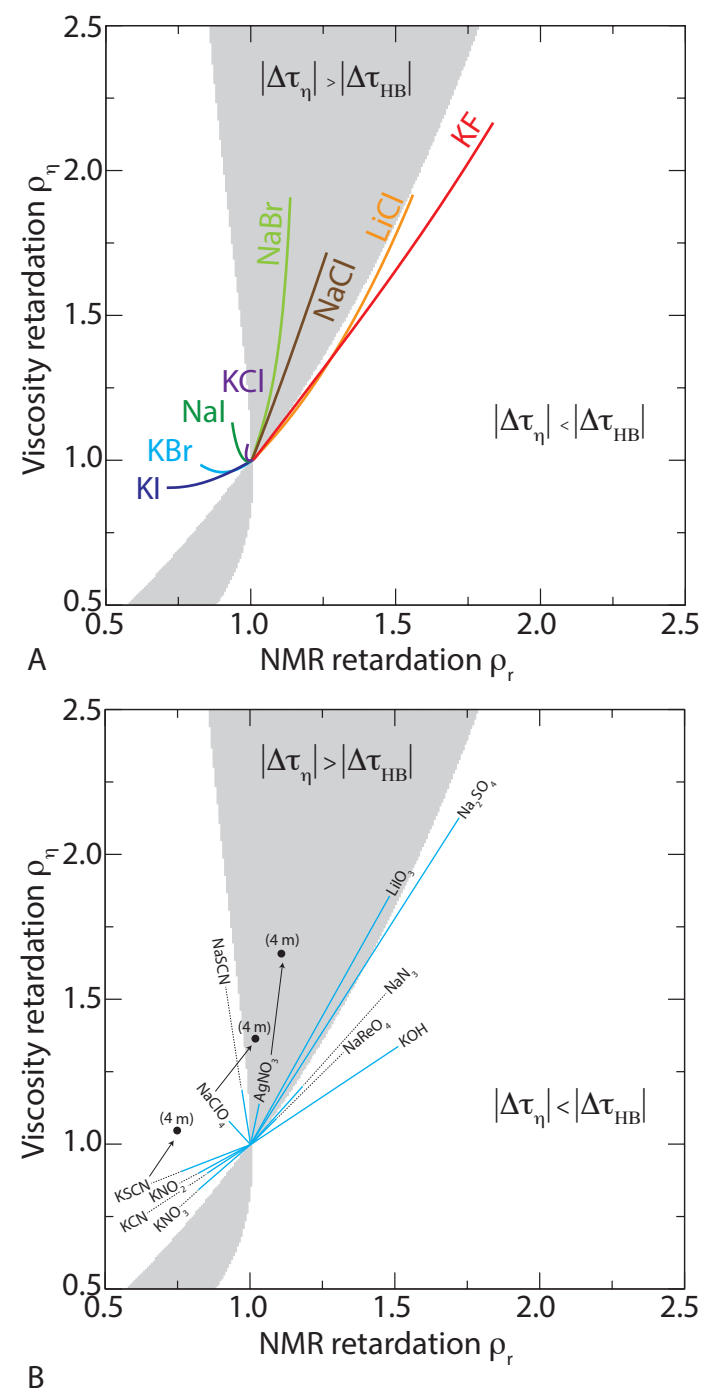

Figure 7: (A) Correlation between the experimental viscosity and the reorientation dynamics of alkali halides (data adapted from Refs. ${ }^{2}$ and $\left.^{38}\right)$. The relative scaling of the viscosity ${ }^{38}$ $\rho_{\eta}$ is plotted against the scaling of the NMR reorientation time ${ }^{2} \rho_{r}$ as concentration increases for all investigated salts (data identical to that of Fig. 1). The grey region corresponds to combinaison of $\rho_{r}$ and $\rho_{\eta}$ such that $\left|\Delta \tau_{\eta}\right|>\left|\Delta \tau_{\mathrm{HB}}\right|$. (B) Similar data for a molecular anion salts, except that the concentration dependence up to $3-\mathrm{m}$ was inferred from the $B_{\eta}$ and $B_{r}$ values. Deviations from linearity as concentration increases are shown by plotting the point at $4 \mathrm{~m}$ for salts for which the concentrationdependence of both $\rho_{r}$ and $\rho_{\eta}$ were available $\left(\mathrm{NaClO}_{4},{ }^{58} \mathrm{KSCN}^{59}\right.$ and $\left.\mathrm{AgNO}_{3}{ }^{60}\right)$.

creases. 
In order to provide a molecular picture of the underlying mechanisms for the effect of ions on these different aspects of water dynamics, we used molecular dynamics simulations with non-polarizable, classical forcefields. Previous studies showed that typical non-polarizable ion forcefields fail to reproduce the acceleration of water dynamics observed experimentally for some salts, and that they always lead to a slowdown of dynamics. ${ }^{14,15}$ We have thus chosen to consider the ECCR approach ${ }^{18-22}$ for the ion forcefield, where all ion charges are scaled by 0.75 to implicitly account for the fast electronic contribution to the dielectric response of water, and where the ion diameters are slightly rescaled. We generalized the approach used in refs. ${ }^{19-22}$ to extend it to the entire series of halide and alkali ions.

In contrast with full charge models, the ECCR approach is shown to lead to results in remarkable agreement with experiments, as suggested previously. ${ }^{15,28}$ In particular, reorientational and translational dynamics are indeed accelerated in solutions of structure-breaking salts, while they can be strongly retarded for structure-breakers. The trends observed in the experiments among the halide and the alkali series is fully reproduced by the simulations, and the agreement is quantitative in many cases. They thus appear as a successful and computationally-cheap alternative to more elaborate polarizable ${ }^{61}$ or ab-initio ${ }^{39}$ descriptions. However, limitations still appear for the alkali cations, with the current approach failing to reproduce the very large retardation induced by the lithium ion and to some extent, by sodium. The importance of charge-transfer between the ions and water, which can be explicitly taken into account into more elaborate models, could help to solve these discrepancies. $^{40}$

An additional key feature of the ECCR forcefields was to correctly predict the trends of the translational-rotational decoupling (Fig. 3) among the different salts. The reasons for the observed variations remain unclear and would deserve future attention.

Provided with a reliable set of simulation results, we have decomposed the water long- time reorientation dynamics into its two components, i.e., the HB exchange through large amplitude jumps and the slower reorientation of the frame of an intact HB between the jumps, which scales as the solution viscosity. As already suggested in our previous studies on other ions, ${ }^{5,7,13}$ the jumps are a key factor to determine whether an isolated ion accelerates or decelerates water dynamics. In particular, the strength of HBs that can form between an anion and a water $\mathrm{OH}$ group greatly varies among the halide series, and explains for example why water reorientation and translation dynamics is accelerated in the presence of dilute $\mathrm{I}^{-}$but strongly decelerates in solutions that contain $\mathrm{F}^{-}$.

At higher concentrations, the frame reorientation, that correlates with the solution viscosity, ${ }^{7,12}$ slows down as compared to the dilute cases. However, HB exchanges always remain the main reorientation pathways, accounting in most cases for two thirds of water reorientation. As a consequence, any change in the jump time has a much more pronounced effect on the reorientation dynamics than has a change in the solution viscosity. In particular, the increased viscosity in some strongly accelerating solutions shows that the dynamics is dominated by the HB exchanges, that are accelerated in these cases because of weaker HBs with the anions.

Finally, we have shown that one can infer from experimental data the respective contributions of $\mathrm{HB}$ exchanges and of viscosity to water reorientation dynamics in salt solutions. This approach is thus free from any limitation of the MD simulation forcefields. No unique scenario regarding the dominant effect of $\mathrm{HB}$ exchanges or viscosity on the overall retardation is found. Out of the 20 examined salts, the faster or slower HB exchange dynamics brings the dominant contribution up to high concentrations for two thirds of them. In the other cases, the increased viscosity in concentrated solutions is responsible for the slower dynamics, as already suggested in a previous study on one of these salts, ${ }^{7}$ but which does not represent a general explanation for the slowdown in all salt solutions.

The diagram shown in Fig. 7 can be plotted 
for any salts for which the NMR reorientation time and viscosity are experimentally known, and can thus be used to determine the dominant cause of water acceleration/deceleration in a given salt solution, without the need of MD simulations. Indeed, it could be useful to know whether the solution affects water reorientations dynamics mainly because of accelerated/slowed HB exchanges, or to a change in viscosity. This could help optimize e.g. the proton transfer/mobility, which is expected to be more sensitive to HB switches as compared to viscosity.

Acknowledgement The research leading to these results has received funding from the European Research Council under the European Union's Eighth Framework Program (H2020/2014-2020)/ERC Grant Agreement No. 757111 (G.S.). This work was also supported by the "Initiative d'Excellence" program from the French State (Grant "DYNAMO", ANR11-LABX-0011-01 to G.S.).

\section{References}

(1) Marcus, Y. Effect of Ions on the Structure of Water: Structure Making and Breaking. Chem. Rev. 2009, 109, 1346-1370.

(2) Endom, L.; Hertz, H. G.; Thül, B.; Zeidler, M. D. A Microdynamic Model of Electrolyte Solutions As Derived from Nuclear Magnetic Relaxation and SelfDiffusion Data. Ber. Bunsenges. Phys. Chem. 1967, 71, 1008-1228.

(3) Laage, D.; Stirnemann, G.; Sterpone, F.; Rey, R.; Hynes, J. T. Reorientation and Allied Dynamics in Water and Aqueous Solutions. Annu. Rev. Phys. Chem. 2011, 62, 395-416.

(4) Holzmann, J.; Ludwig, R.; Geiger, A.; Paschek, D. Pressure and Salt Effects in Simulated Water: Two Sides of the Same Coin? Angew. Chem. Int. Ed. Engl. 2007, 46, 8907-8911.

(5) Boisson, J.; Stirnemann, G.; Laage, D.; Hynes, J. T. Water Reorientation Dynam- ics in the First Hydration Shells of F- and I-. Phys. Chem. Chem. Phys. 2011, 13, 19895-19901.

(6) Laage, D.; Hynes, J. T. Reorientional Dynamics of Water Molecules in Anionic Hydration Shells. Proc. Natl. Acad. Sci. U.S.A. 2007, 104, 11167-11172.

(7) Stirnemann, G.; Wernersson, E.; Jungwirth, P.; Laage, D. Mechanisms of Acceleration and Retardation of Water Dynamics by Ions. J. Am. Chem. Soc. 2013, 135, 11824-11831.

(8) Laage, D.; Hynes, J. T. A Molecular Jump Mechanism of Water Reorientation. Science 2006, 311, 832-835.

(9) Laage, D.; Stirnemann, G.; Hynes, J. T. Why Water Reorientation Slows Without Iceberg Formation Around Hydrophobic Solutes. J. Phys. Chem. B 2009, 113, 2428-2435.

(10) Sterpone, F.; Stirnemann, G.; Hynes, J. T.; Laage, D. Water HydrogenBond Dynamics Around Amino Acids: The Key Role of Hydrophilic HydrogenBond Acceptor Groups. J. Phys. Chem. B 2010, 114, 2083-2089.

(11) Stirnemann, G.; Hynes, J. T.; Laage, D. Water Hydrogen Bond Dynamics in Aqueous Solutions of Amphiphiles. J. Phys. Chem. B 2010, 114, 3052-3059.

(12) Zhang, Q.; Wu, T.; Chen, C.; Mukamel, S.; Zhuang, W. Molecular Mechanism of Water Reorientational Slowing down in Concentrated Ionic Solutions. Proc. Natl. Acad. Sci. U.S.A. 2017, 114, 10023-10028.

(13) Stirnemann, G.; Jungwirth, P.; Laage, D. Water Dynamics in Concentrated Electrolytes: Local Ion Effect on HydrogenBond Jumps Rather Than Collective Coupling to Ion Clusters. Proc. Natl. Acad. Sci. U.S.A. 2018, 115, E4953-E4954. 
(14) Kim, J. S.; Wu, Z.; Morrow, A. R.; Yethiraj, A.; Yethiraj, A. Self-Diffusion and Viscosity in Electrolyte Solutions. J. Phys. Chem. B 2012, 116, 12007-12013.

(15) Kann, Z. R.; Skinner, J. L. A ScaledIonic-Charge Simulation Model That Reproduces Enhanced and Suppressed Water Diffusion in Aqueous Salt Solutions. J. Chem. Phys. 2014, 141, 104507.

(16) Lopes, P. E.; Roux, B.; MacKerell, A. D. Molecular Modeling and Dynamics Studies with Explicit Inclusion of Electronic Polarizability: Theory and Applications. Theo. Chem. Acc. 2009, 124, 11-28.

(17) Annapureddy, H. V.; Dang, L. X. Understanding the Rates and Molecular Mechanism of Water-Exchange Around Aqueous Ions Using Molecular Simulations. J. Phys. Chem. B 2014, 118, 8917-8927.

(18) Leontyev, I.; Stuchebrukhov, A. Accounting for Electronic Polarization in NonPolarizable Force Fields. Phys. Chem. Chem. Phys. 2011, 13, 2613-2626.

(19) Mason, P. E.; Wernersson, E.; Jungwirth, P. Accurate Description of Aqueous Carbonate Ions: An Effective Polarization Model Verified by Neutron Scattering. J. Phys. Chem. B 2012, 116, 8145-8153.

(20) Pluharová, E.; Mason, P. E.; Jungwirth, P. Ion Pairing in Aqueous Lithium Salt Solutions with Monovalent and Divalent Counter-Anions. J. Phys. Chem. A 2013, 117, 11766-11773.

(21) Pluharová, E.; Fischer, H. E.; Mason, P. E.; Jungwirth, P. Hydration of the Chloride Ion in Concentrated Aqueous Solutions Using Neutron Scattering and Molecular Dynamics. Mol. Phys. 2014, 112, 1230-1240.

(22) Kohagen, M.; Mason, P. E.; Jungwirth, P. Accounting for Electronic Polarization Effects in Aqueous Sodium Chloride Via Molecular Dynamics Aided by Neutron
Scattering. J. Phys. Chem. B 2016, 120, 1454-1460.

(23) Plimpton, S. Fast Parallel Algorithms for Short-Range Molecular Dynamics. J. Comput. Chem. 1995, 117, 1-19.

(24) Berendsen, H. J. C.; Grigera, J. R.; Straatsma, T. P. The Missing Term in Effective Pair Potentials. J. Phys. Chem. 1987, 91, 6269-6271.

(25) Ryckaert, J. P.; Ciccotti, G.; Berendsen, H. J. Numerical Integration of the Cartesian Equations of Motion of a System with Constraints: Molecular Dynamics of N-Alkanes. J. Comput. Phys. 1977, 23, 327-341.

(26) Martinez, L.; Andrade, R.; Birgin, E. G.; Martínez, J. M. PACKMOL: A Package for Building Initial Configurations for Molecular Dynamics Simulations. J. Comput. Chem. 2009, 30, 2157-2164.

(27) Reiser, S.; Horsch, M.; Hasse, H. Temperature Dependence of the Density of Aqueous Alkali Halide Salt Solutions by Experiment and Molecular Simulation. J. Chem. Eng. Data 2014, 59, 3434-3448.

(28) Benavides, A. L.; Portillo, M. A.; Chamorro, V. C.; Espinosa, J. R.; Abascal, J. L.; Vega, C. A Potential Model for Sodium Chloride Solutions Based on the TIP4P/2005 Water Model. J. Chem. Phys. 2017, 14\%, 104501.

(29) Horinek, D.; Mamatkulov, S. I.; Netz, R. R. Rational Design of Ion Force Fields Based on Thermodynamic Solvation Properties. J. Chem. Phys. 2009, 130, 124507.

(30) Soper, A. K.; Weckström, K. Ion Solvation and Water Structure in Potassium Halide Aqueous Solutions. Biophys. Chem. 2006, 124, 180-191.

(31) Northrup, S. H.; Hynes, J. T. The Stable States Picture of Chemical Reactions. I. Formulation for Rate Constants and 
Initial Condition Effects. J. Chem. Phys. 1980, 73, 2700-2714.

(32) Bakker, H. J.; Skinner, J. L. Vibrational Spectroscopy As a Probe of Structure and Dynamics in Liquid Water. Chem. Rev. 2010, 110, 1498-1517.

(33) Bagno, A.; Rastrelli, F.; Saielli, G. NMR Techniques for the Investigation of Solvation Phenomena and Non-Covalent Interactions. Prog. Nucl. Mag. Res. Sp. 2005, $47,41-93$.

(34) Heugen, U.; Schwaab, G.; Brundermann, E.; Heyden, M.; Yu, X.; Leitner, D. M.; Havenith, M. Solute-Induced Retardation of Water Dynamics Probed Directly by Terahertz Spectroscopy. Proc. Natl. Acad. Sci. U.S.A. 2006, 103, 1230112306.

(35) Tielrooij, K. J.; Paparo, D.; Piatkowski, L.; Bakker, H. J.; Bonn, M. Dielectric Relaxation Dynamics of Water in Model Membranes Probed by Terahertz Spectroscopy. Biophys. J. 2009, 97, 2484-2492.

(36) Winkler, K.; Lindner, J.; Bürsing, H.; Vöhringer, P. Ultrafast Raman-Induced Kerr-Effect of Water: Single Molecule Versus Collective Motions. J. Chem. Phys. 2000, 113, 4674-4682.

(37) Donald, H.; Jenkins, B.; Marcus, Y. Viscosity S-Coefficients of Ions in Solution. Chem. Rev. 1995, 95, 2695-2724.

(38) Goldsack, D. E.; Franchetto, R. the Viscosity of Concentrated Electrolyte Solutions. I. Concentration Dependence at Fixed Temperature. Can. J. Chem. 1977, 55, 1062-1072.

(39) Ding, Y.; Hassanali, A. A.; Parrinello, M. Anomalous Water Diffusion in Salt Solutions. Proc. Natl. Acad. Sci. U.S.A. 2014, 111, 3310-3315.
(40) Yao, Y.; Berkowitz, M. L.; Kanai, Y. Communication: Modeling of Concentration Dependent Water Diffusivity in Ionic Solutions: Role of Intermolecular Charge Transfer. J. Chem. Phys. 2015, 143, 241101.

(41) Müller, K. J.; Hertz, H. G. A Parameter As an Indicator for Water?Water Association in Solutions of Strong Electrolytes. J. Phys. Chem. 1996, 100, 1256-1265.

(42) Stirnemann, G.; Sterpone, F.; Laage, D. Dynamics of Water in Concentrated Solutions of Amphiphiles: Key Roles of Local Structure and Aggregation. J. Phys. Chem. B 2011, 115, 3254-3262.

(43) Stirnemann, G.; Duboué-Dijon, E.; Laage, D. Ab Initio Simulations of Water Dynamics in Aqueous TMAO Solutions: Temperature and Concentration Effects. J. Phys. Chem. B 2017, 121, 11189-11197.

(44) Duboué-Dijon, E.; Fogarty, A. C.; Hynes, J. T.; Laage, D. Dynamical Disorder in the DNA Hydration Shell. J. Am. Chem. Soc. 2016, 138, 7610-7620.

(45) Sterpone, F.; Stirnemann, G.; Laage, D. Magnitude and Molecular Origin of Water Slowdown Next to a Protein. J. Am. Chem. Soc. 2012, 134, 4116-4119.

(46) Stirnemann, G.; Rossky, P. J.; Hynes, J. T.; Laage, D. Water Reorientation, Hydrogen-Bond Dynamics and 2D-IR Spectroscopy Next to an Extended Hydrophobic Surface. Faraday Discuss. 2010, 146, 263-281.

(47) Stirnemann, G.; Castrillón, S. R. V.; Hynes, J. T.; Rossky, P. J.; Debenedetti, P. G.; Laage, D. NonMonotonic Dependence of Water Reorientation Dynamics on Surface Hydrophilicity: Competing Effects of the Hydration Structure and Hydrogen-Bond Strength. Phys. Chem. Chem. Phys. 2011, 13, 19911-19917. 
(48) Xiao, S.; Figge, F.; Stirnemann, G.; Laage, D.; McGuire, J. A. Orientational Dynamics of Water at an Extended Hydrophobic Interface. J. Am. Chem. Soc. 2016, 138, 5551-5560.

(49) Fogarty, A. C.; Duboué-Dijon, E.; Laage, D.; Thompson, W. H. Origins of the Non-Exponential Reorientation Dynamics of Nanoconfined Water. $J$. Chem. Phys. 2014, 141, 18C523.

(50) Stirnemann, G.; Laage, D. Communication: On the Origin of the Non-Arrhenius Behavior in Water Reorientation Dynamics. J. Chem. Phys. 2012, 137, 031101.

(51) Kincaid, J. F.; Eyring, H.; Stearn, A. E. the Theory of Absolute Reaction Rates and Its Application to Viscosity and Diffusion in the Liquid State. Chem. Rev. 1941, 28, 301-365.

(52) Bajaj, P.; Richardson, J. O.; Paesani, F. Ion-Mediated Hydrogen-Bond Rearrangement Through Tunnelling in the IodideDihydrate Complex. Nat. Chem. 2019, doi 10.1038/s41557-019-0220-2.

(53) Wilkins, D. M.; Manolopoulos, D. E.; Pipolo, S.; Laage, D.; Hynes, J. T. Nuclear Quantum Effects in Water Reorientation and Hydrogen-Bond Dynamics. J. Phys. Chem. Lett. 2017, 8, 2602-2607.

(54) Berkelbach, T. C.; Lee, H. S.; Tuckerman, M. E. Concerted Hydrogen-Bond Dynamics in the Transport Mechanism of the Hydrated Proton: A First-Principles Molecular Dynamics Study. Phys. Rev. Lett. 2009, 103, 238302.

(55) Fayer, M. D.; Moilanen, D. E.; Wong, D.; Rosenfeld, D. E.; Fenn, E. E.; Park, S. Water Dynamics in Salt Solutions Studied with Ultrafast Two-Dimensional Infrared (2D IR) Vibrational Echo Spectroscopy. Acc. Chem. Res. 2009, 42, 1210-1219.

(56) Ji, M.; Odelius, M.; Gaffney, K. J. Large Angular Jump Mechanism Observed for Hydrogen Bond Exchange in Aqueous
Perchlorate Solution. Science 2010, 328, 1003-1005.

(57) Laage, D.; Stirnemann, G.; Sterpone, F.; Hynes, J. T. Water Jump Reorientation: From Theoretical Prediction to Experimental Observation. Acc. Chem. Res. 2012, 45, 53-62.

(58) Miller, M. L.; Doran, M. Concentrated Salt Solutions. II. Viscosity and Density of Sodium Thiocyanate, Sodium Perchlorate and Sodium Iodide. J. Phys. Chem. 1956, 60, 186-189.

(59) Rohman, N.; Dass, N. N.; Mahiuddin, S. Speeds of Sound and Viscosities of Potassium Thiocyanate in Water, Methanol, and Propylene Carbonate. Aust. J. Chem. 2000, 53, 463-469.

(60) Jones, G.; Colvin, J. H. The Viscosity of Solutions of Electrolytes As a Function of the Concentration. VII. Silver Nitrate, Potassium Sulfate and Potassium Chromate. J. Am. Chem. Soc. 1940, 62, 338340 .

(61) Grossfield, A.; Ren, P.; Ponder, J. W. Ion Solvation Thermodynamics from Simulation with a Polarizable Force Field. J. Am. Chem. Soc. 2003, 125, 15671-15682. 


\section{Biographical Information}

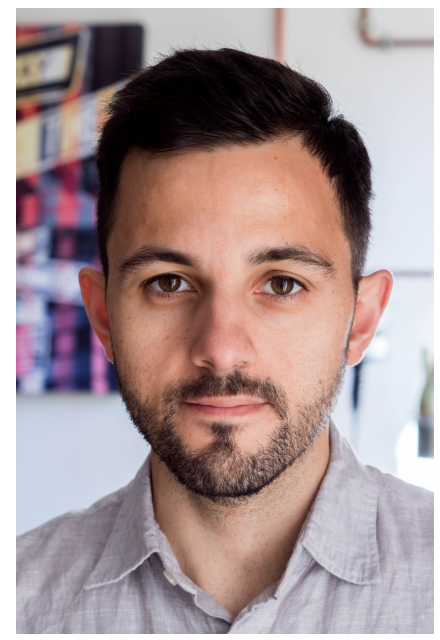

Guillaume Stirnemann was born in France in 1987. After undergraduate studies at the École normale supérieure (ENS) and a Master's thesis with Pablo Debenedetti at Princeton University, he received his Ph. D. (2011) from ENS and Sorbonne Université (Paris, France), under the supervision of Damien Laage. He was then a postdoctoral fellow at Columbia University with Bruce Berne and later at the Institut de Biologie Physico-Chimique (IBPC) in Paris with Fabio Sterpone. In 2014, he was recruited as a CNRS researcher at IBPC where the current interests of his group include the stability, the mechanical properties and the reactivity of biomolecules, and transport phenomena in aqueous solutions, with a special emphasis on questions related to the origins of life. 
Graphical TOC Entry

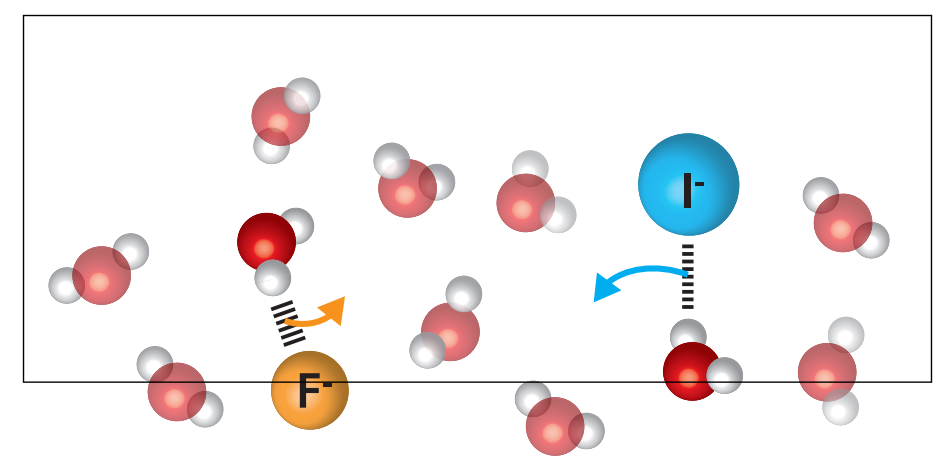

